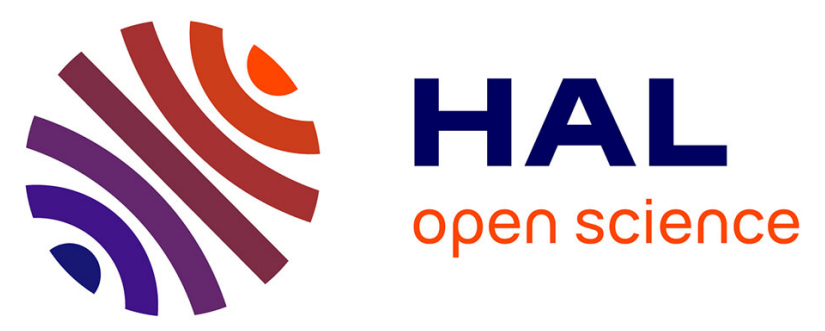

\title{
Analytical model for the optical functions of amorphous semiconductors from the near-infrared to ultraviolet: Applications in thin film photovoltaics
}

\author{
A. S. Ferlauto, G. M. Ferreira, J. M. Pearce, C. R. Wronski, R. W. Collins, \\ Xunming Deng, Gautam Ganguly
}

\section{To cite this version:}

A. S. Ferlauto, G. M. Ferreira, J. M. Pearce, C. R. Wronski, R. W. Collins, et al.. Analytical model for the optical functions of amorphous semiconductors from the near-infrared to ultraviolet: Applications in thin film photovoltaics. Journal of Applied Physics, 2002, 92 (5), pp.2424-2436. 10.1063/1.1497462 . hal-02120549

\section{HAL Id: hal-02120549 \\ https://hal.science/hal-02120549}

Submitted on 6 May 2019

HAL is a multi-disciplinary open access archive for the deposit and dissemination of scientific research documents, whether they are published or not. The documents may come from teaching and research institutions in France or abroad, or from public or private research centers.
L'archive ouverte pluridisciplinaire HAL, est destinée au dépôt et à la diffusion de documents scientifiques de niveau recherche, publiés ou non, émanant des établissements d'enseignement et de recherche français ou étrangers, des laboratoires publics ou privés. 


\title{
Analytical model for the optical functions of amorphous semiconductors from the near-infrared to ultraviolet: Applications in thin film photovoltaics
}

\author{
A. S. Ferlauto, G. M. Ferreira, J. M. Pearce, C. R. Wronski, and R. W. Collins ${ }^{\text {a) }}$ \\ Department of Physics, Materials Research Institute, and Center for Thin Film Devices, \\ The Pennsylvania State University, University Park, Pennsylvania 16802 \\ Xunming Deng \\ Department of Physics and Astronomy, University of Toledo, Toledo, Ohio 43606 \\ Gautam Ganguly \\ BP Solar, 3601 LaGrange Parkway, Toano, Virginia 23168
}

(Received 16 April 2002; accepted for publication 7 June 2002)

\begin{abstract}
We have developed a Kramers-Kronig consistent analytical expression to fit the measured optical functions of hydrogenated amorphous silicon $(a-\mathrm{Si}: \mathrm{H})$ based alloys, i.e., the real and imaginary parts of the dielectric function $\left(\epsilon_{1}, \epsilon_{2}\right)$ (or the index of refraction $n$ and absorption coefficient $\alpha$ ) versus photon energy $E$ for the alloys. The alloys of interest include amorphous silicon-germanium $\left(a-\mathrm{Si}_{1-x} \mathrm{Ge}_{x}: \mathrm{H}\right)$ and silicon-carbon $\left(a-\mathrm{Si}_{1-x} \mathrm{C}_{x}: \mathrm{H}\right)$, with band gaps ranging continuously from $\sim 1.30$ to $1.95 \mathrm{eV}$. The analytical expression incorporates the minimum number of physically meaningful, $E$ independent parameters required to fit $\left(\epsilon_{1}, \epsilon_{2}\right)$ versus $E$. The fit is performed simultaneously throughout the following three regions: (i) the below-band gap (or Urbach tail) region where $\alpha$ increases exponentially with $E$, (ii) the near-band gap region where transitions are assumed to occur between parabolic bands with constant dipole matrix element, and (iii) the above-band gap region where $\left(\epsilon_{1}, \epsilon_{2}\right)$ can be simulated assuming a single Lorentz oscillator. The expression developed here provides an improved description of $\epsilon_{2}$ (or $\alpha$ ) in the below-band gap and near-band gap regions compared with previous approaches. Although the expression is more complicated analytically, it has numerous applications in the analysis and simulation of thin film $a$-Si:H based $p-i-n$ and $n-i-p$ multilayer photovoltaic devices. First, we describe an approach whereby, from a single accessible measure of the optical band gap, the optical functions can be generated over the full solar spectrum for a sample set consisting of the highest quality intrinsic $a$-Si:H based alloys prepared by plasma-enhanced chemical vapor deposition using the principle of maximal $\mathrm{H}_{2}$ dilution. Second, we describe quantitatively how such an approach can be modified for sample sets consisting of lower quality alloy materials. Finally, we demonstrate how the generated optical functions can be used in simulations of the absorption, reflection, and quantum efficiency spectra of $a$-Si:H based single-junction and multijunction solar cells. (C) 2002 American Institute of Physics. [DOI: 10.1063/1.1497462]
\end{abstract}

\section{INTRODUCTION}

Analytical expressions for the optical functions of thin film semiconductors are useful for two important purposes, namely, materials characterization ${ }^{1}$ and device performance simulation. $^{2}$ Such expressions describe either the real and imaginary parts of the dielectric function $\left(\epsilon_{1}, \epsilon_{2}\right)$ versus photon energy $E$ or the index of refraction $n$ and absorption coefficient $\alpha$ versus $E$.

First, such expressions can be applied in the analysis of optical data including transmittance, reflectance, and ellipsometric spectra obtained on the thin film semiconductors. The conventional analysis approach for such data is to apply mathematical inversion (either analytically or numerically) and extract the optical functions along with the overall film thickness. ${ }^{3,4}$ In some cases, the microscopic surface rough-

\footnotetext{
${ }^{a)}$ Electronic mail: rwc6@psu.edu
}

ness layer thickness can also be extracted. ${ }^{4}$ From the dependence of $\left(\epsilon_{1}, \epsilon_{2}\right)$ [or $\left.(n, \alpha)\right]$ on $E$ in the region just above the absorption onset, a measure of the optical band gap $E_{g}$ is obtained. Because of the high sensitivity of the optical functions to the thin film preparation technique, this procedure must be applied routinely on a sample-by-sample basis to extract key characteristics necessary for the assessment of materials and fabrication methods. With a reliable expression for the optical functions in terms of photon energy independent parameters, however, the analysis of such data can proceed by least-squares regression rather than by mathematical inversion. ${ }^{5}$ As a result, physically significant parameters are a direct outcome of the analysis, including: (i) the parameters that describe the optical functions (the most important being the optical gap), (ii) the thicknesses, and (iii) the parameter confidence limits and correlation coefficients.

Second, the analytical expressions can also be used in performance simulations of optoelectronic devices such as 
solar cells. In this application, the device designer specifies as input the photon energy independent parameters such as the optical gap, Urbach tail slope, or even the void volume fraction that describe each of the material components of the device. ${ }^{6}$ These input parameters are then used to calculate the optical functions of the components which in turn are applied in multilayer optical simulations to predict the transmittance (if any), reflectance, absorbance, and optical quantum efficiency spectra for specific device configurations. The designer then adjusts the component material input parameters to predict the effect of incorporating different types of materials into the device.

Attempts of varying success have been applied over the past 15 or more years to develop broadly useful analytical expressions for the optical properties of amorphous semiconductors. ${ }^{7-12}$ The materials of greatest interest have been the hydrogenated group IV thin films including amorphous carbon $(a-\mathrm{C}: \mathrm{H})$, silicon $(a-\mathrm{Si}: \mathrm{H})$, and germanium ( $a-\mathrm{Ge}: \mathrm{H})$; as well as the $a-\mathrm{Si}: \mathrm{H}$ based alloys $\left(a-\mathrm{Si}_{1-x} \mathrm{C}_{x}: \mathrm{H}\right.$ and $\left.a-\mathrm{Si}_{1-x} \mathrm{Ge}_{x}: \mathrm{H}\right)$; and the $\mathrm{Si}$ based oxides and nitrides $\left(a-\mathrm{SiO}_{x}: \mathrm{H}\right.$ and $\left.a-\mathrm{SiN}_{x}: \mathrm{H}\right)$. The materials most useful in devices are prepared by chemical vapor deposition (CVD) and plasma-enhanced CVD (PECVD) and have many applications in large area electronics. ${ }^{2,13,14}$

The first attempt was made by Forouhi and Bloomer (FB), who developed an expression for the index of refraction $n(E)$ and extinction coefficient $k(E)=\hbar c \alpha(E) / 2 E$, versus energy $E$ that includes a total of five parameters. ${ }^{7}$ [In the equation for $k(E), c$ is the speed of light and $h=2 \pi \hbar$ is Planck's constant.] Although the FB expression was found to provide reasonable fits for some amorphous semiconductors, the fits for narrow gap materials such as $a-\mathrm{C}: \mathrm{H}$ containing threefold coordinated $\left(s p^{2}\right) \mathrm{C}$ sometimes yielded unphysical results, i.e., negative optical gaps. ${ }^{15}$

More recently Jellison and Modine (JM) outlined the deficiencies of the FB approach in detail. ${ }^{10,16}$ These include a parabolically increasing $k(E)$ with decreasing energy below the gap to $E=0$ and an incorrect treatment of the KramersKronig transformation from $k(E)$ to $n(E)$. To rectify these problems, JM developed the Tauc-Lorentz expression ${ }^{10}$ in which the imaginary part of the dielectric function $\epsilon_{2}(E)$ $=2 n(E) k(E)$ is given as the product of the Tauc law function ${ }^{17} G(E) \propto\left[\left(E-E_{g}\right)^{2} / E^{2}\right]$ and the Lorentz oscillator function ${ }^{18,19} L(E)$ [see Eq. (1b)] in an attempt to obtain suitable near-gap and above-gap optical responses, respectively. The real part of the dielectric function $\epsilon_{1}(E)=n^{2}(E)$ $-k^{2}(E)$ is obtained as a correct Kramers-Kronig transformation of $\epsilon_{2}(E)$. The Tauc-Lorentz expression has led to improved fits of ellipsometric spectra for $a-\mathrm{Si}: \mathrm{H}$ in comparison to the FB expressions, and currently represents the most widely used parametrization of the optical functions of amorphous semiconductors. In spite of its successes, the TaucLorentz expression has three limitations whose impacts have yet to be fully appreciated and assessed.

First, it was demonstrated by Cody et al. ${ }^{3}$ much earlier that the shape of the interband absorption onset for $a-\mathrm{Si}: \mathrm{H}$ is closely consistent with a formula derived on the assumption of parabolic bands and a constant dipole matrix element, i.e., $\epsilon_{2}(E) \propto\left(E-E_{g}\right)^{2}$. In contrast, the Tauc law formula was de- rived on the assumption of parabolic bands and a constant momentum matrix element, i.e., $\epsilon_{2}(E) \propto\left[\left(E-E_{g}\right)^{2} / E^{2}\right]$. Photoemission spectroscopy undertaken by Jackson et al. ${ }^{20}$ was also found to be more closely consistent with a constant dipole matrix element. The net effect of this situation is that optical gap determinations via the Tauc plot, i.e., linear extrapolations of $\left[E^{2} \epsilon_{2}(E)\right]^{1 / 2}$ to zero ordinate, depend on the accessible range of $\epsilon_{2}$ in the measurement. For example, from a reflection measurement such as spectroscopic ellipsometry (SE), it is more difficult to obtain accurate $\epsilon_{2}$ spectra at low values (i.e., for $E \approx E_{g}$ ) due to uncertainties associated with the optical nature of the surface. Thus, when such a measurement is limited to higher $\epsilon_{2}$ values, it provides a relatively wide band gap via linear extrapolation owing to the upward curvature of the Tauc plot versus $E$. In contrast, the band gap is narrower when obtained using lower $\epsilon_{2}$ values, which usually can be obtained more accurately from transmission measurements.

Second, weak exponentially increasing absorption with increasing $E$, i.e., an Urbach tail, exists below the band gap in amorphous semiconductors due to the disorder of the amorphous network. ${ }^{3}$ Although the Urbach tail is not normally measured by SE, due to the sensitivity limitations noted in the previous paragraph, this tail does appear clearly in photothermal, photoconductivity, and transmittance spectra (the latter on thick films) due to their direct sensitivity to $\epsilon_{2}(E)$ on a logarithmic scale. For the JM model, however, $\epsilon_{2}(E)$ is assumed to vanish below the band gap, and so has limitations in the near-gap region, especially when optical functions from transmittance and ellipsometric spectra are spliced together and fitted.

Third, in our experiences in fitting $a$-Si:H based alloys, the constant contribution $\epsilon_{1 \infty}$ to the real part of the dielectric function (see Sec. II) deduced from the Tauc-Lorentz expression varies from above unity for the wide band gap $a-\mathrm{Si}_{1-x} \mathrm{C}_{x}: \mathrm{H}$ alloys to less than unity for pure $a-\mathrm{Si}: \mathrm{H}$, and finally to near and below zero for the $a-\mathrm{Si}_{1-x} \mathrm{Ge}_{x}: \mathrm{H}$ alloys. ${ }^{12}$ In theory, however, $\epsilon_{1 \infty}$ should be either unity if all electronic transitions are included in the model for $\epsilon_{2}(E)$, or greater than unity if sets of transitions exist in $\epsilon_{2}(E)$ above the upper energy limit of the data that are not included explicitly in the model for $\epsilon_{2}(E)$. Evidently one or more ingredients are missing from the Tauc-Lorentz expression. For example, with this expression, the energy above which the near-gap Tauc behavior can be neglected is a rather large fixed multiple of the optical band gap, whereas the situation in reality may require more flexibility.

\section{PARAMETRIC MODELING}

In this part, we describe the two simple modifications of the Tauc-Lorentz formula that allow us to enhance the capabilities of the analytical model for the optical functions of $a-\mathrm{Si}: \mathrm{H}$ based alloys. We start by writing the imaginary part of the dielectric function as 


$$
\epsilon_{2}(E)= \begin{cases}\frac{E_{1}}{E} \exp \left\{\frac{\left(E-E_{t}\right)}{E_{u}}\right\} ; & 0<E \leqslant E_{t} ; \\ G(E) L(E)=G(E) \frac{A E_{0} \Gamma E}{\left[\left(E^{2}-E_{0}^{2}\right)^{2}+\Gamma^{2} E^{2}\right]} ; & E>E_{t},\end{cases}
$$

where $E_{t}$ is the demarcation energy between the Urbach tail transitions and the band-to-band transitions. In Eq. (1a), the expression for $0<E \leqslant E_{t}$ leads to the Urbach form of the absorption coefficient, $\alpha(E)=\alpha_{0} \exp \left(E / E_{u}\right)$, neglecting the variation in $\epsilon_{1}(E)$ over the range of energy $0<E \leqslant E_{t}$. In Eq. (1b) the expression for $E>E_{t}$ includes the product of two terms, the Lorentz oscillator function $L(E)$ and a variable band edge function $G(E)$. $G(E)$ forces $\epsilon_{2}(E)$ to assume a desired form for energies just above the optical gap $E_{g}$. In addition, $G(E) \rightarrow 1$ for $E \gg E_{g}$ so that the desired result $\epsilon_{2}(E) \rightarrow L(E)$ holds at high energies. In Eq. (1a), $E_{1}$ is defined so that $\epsilon_{2}(E)$ is continuous at $E=E_{t}$; thus

$$
E_{1}=E_{t} L\left(E_{t}\right) G\left(E_{t}\right) .
$$

Finally, in Eq. (1b) for $L(E),\left(A, E_{0}, \Gamma\right)$ denote the Lorentz oscillator amplitude, resonance energy, and oscillator width, respectively. ${ }^{18,19}$ Equations (1) generalize the approach first described by JM in that the Urbach tail is added and the function $G(E)$ can now be selected for consistency with the observed shape of the absorption onset.

The real part $\epsilon_{1}(E)$ is determined as usual from a Kramers-Kronig transformation

$$
\begin{aligned}
& \epsilon_{1}(E)=\epsilon_{1 \infty}+I_{U}(E)+I_{L}(E) \\
& =\epsilon_{1 \infty}+\frac{2 E_{1}}{\pi} P \int_{0}^{E_{t}} \frac{\exp \left[\left(E^{\prime}-E_{t}\right) / E_{u}\right]}{E^{\prime 2}-E^{2}} d E^{\prime} \\
& +\frac{2}{\pi} P \int_{E_{t}}^{\infty} \frac{E^{\prime} G\left(E^{\prime}\right) L\left(E^{\prime}\right)}{E^{\prime 2}-E^{2}} d E^{\prime},
\end{aligned}
$$

where " $P$ " denotes the principal values of the integrals. 18,19 $I_{U}(E)$ and $I_{L}(E)$ in Eq. (2a) denote the Urbach tail and Lorentz oscillator integrals appearing as the second and third terms in Eq. (2b), respectively.

Once $\epsilon_{1}(E)$ and $\epsilon_{2}(E)$ are determined from Eqs. (1) and $(2)$, the index of refraction $n(E)$ and absorption coefficient $\alpha(E)$ can be established from

$$
\begin{aligned}
& n=\left\{\left[\left(\epsilon_{1}^{2}+\epsilon_{2}^{2}\right)^{1 / 2}+\epsilon_{1}\right] / 2\right\}^{1 / 2}, \\
& \alpha=(2 E / \hbar c)\left\{\left[\left(\epsilon_{1}^{2}+\epsilon_{2}^{2}\right)^{1 / 2}-\epsilon_{1}\right] / 2\right\}^{1 / 2} .
\end{aligned}
$$

In the following Secs. II A and II B, the constant momentum (Tauc) and constant dipole matrix element forms for $G(E)$ are employed, respectively, and expressions will be provided for $I_{L}(E)$ for these two cases. In Sec. II C, an expression for $I_{U}(E)$ will be provided for completeness, although for $a$-Si:H based materials, this contribution to $\epsilon_{1}(E)$ is very small. In fact, if one desires to eliminate the Urbach tail completely from consideration in the expressions given later, one simply sets $E_{1}=0\left[\right.$ so $\left.I_{U}(E)=0\right]$ and $E_{t}=E_{g}$ in Eq. (2b). Finally, in Sec. II C issues of convergence of the integrals for $E$ near 0 and $E_{t}$ will be discussed briefly for the general case in which $I_{U}(E)$ is included in $\epsilon_{1}(E)$.

\section{A. Determination of $I_{L}(E)$ assuming constant momentum matrix element}

In the Tauc theory of optical absorption, parabolic bands are assumed along with a constant momentum matrix element. ${ }^{17}$ These assumptions lead to variations in $\epsilon_{2}(E)$ and $\alpha(E)$ of the form $\left[\epsilon_{2}(E) E^{2}\right]^{1 / 2} \propto[\alpha(E) n(E) E]^{1 / 2} \propto(E$ $\left.-E_{g}\right)$. In accordance with this proportionality, JM used the following Tauc (" $T$ ") form for $G(E)$ in Eq. (1b): ${ }^{10}$

$$
G_{T}(E)=\frac{\left(E-E_{g}\right)^{2}}{E^{2}} .
$$

A minor complication exists in that, for $E \ll E_{0}$, the Lorentz oscillator expression in Eq. (1b) exhibits the approximate form $L(E) \approx\left(A \Gamma / E_{0}^{3}\right) E$. Thus, in order for the product $\epsilon_{2}(E)=G(E) L(E)$ in Eq. (1b) to follow the Tauc theory of Eq. (4) for $E$ near $E_{g}$, then the $E^{2}$ factor in the denominator of Eq. (4) should be replaced by $E^{3}$. Unfortunately, this violates the requirement that $G_{T}(E) \rightarrow 1$ for $E \gg E_{g}$ and would lead to an expression for $\epsilon_{2}(E)$ that differs from the desired high energy asymptotic form of the Lorentz oscillator, namely $L(E) \propto E^{-3}$. Thus, it should be realized that Eq. (4) sacrifices accuracy in $\epsilon_{2}(E)$ near the optical band gap for overall analytical simplicity.

Substituting Eq. (4) into the third term of Eq. (2b) and setting $I_{T L}=I_{L}(E)$ (where $T$ designates the Tauc expression for $G$ ) yields

$$
\begin{aligned}
I_{T L}\left(a_{i T} ; i=\right. & \left.0,1,2,3 ; c_{0 T}, d_{0 T}\right) \\
= & \frac{2 A E_{0} \Gamma}{\pi}\left[a_{3 T}\left\{\zeta^{2} I_{1 T}-\ln \left[L_{D}\left(E_{t}\right)\right]^{1 / 4}\right\}\right. \\
& +a_{2 T}\left(I_{0 A T}+I_{0 B T}\right)+a_{1 T} I_{1 T}+a_{0 T}\left(\frac{I_{0 A T}-I_{0 B T}}{E_{0}^{2}}\right) \\
& \left.-c_{0 T} \ln \left|E-E_{t}\right|-d_{0 T} \ln \left(E+E_{t}\right)\right],
\end{aligned}
$$

where

$$
\begin{aligned}
& I_{1 T}=\frac{1}{2 \chi \Gamma}\left[\pi-2 \tan ^{-1}\left(2 \frac{E_{t}^{2}-\zeta^{2}}{\chi \Gamma}\right)\right], \\
& I_{0 A T}=\frac{1}{2 \Gamma}\left[\pi-\tan ^{-1}\left(\frac{2 E_{t}+\chi}{\Gamma}\right)+\tan ^{-1}\left(\frac{-2 E_{t}+\chi}{\Gamma}\right)\right], \\
& I_{0 B T}=\frac{1}{4 \chi} \ln \left(\frac{E_{t}^{2}+E_{0}^{2}+\chi E_{t}}{E_{t}^{2}+E_{0}^{2}-\chi E_{t}}\right),
\end{aligned}
$$




$$
\begin{aligned}
& \zeta=\left[E_{0}^{2}-\left(\Gamma^{2} / 2\right)\right]^{1 / 2}, \\
& \chi=\left(4 E_{0}^{2}-\Gamma^{2}\right)^{1 / 2}, \\
& c_{0 T}=\frac{E G_{T}(E)}{2 L_{D}(E)}, \\
& d_{0 T}=\frac{-\left(E+E_{g}\right)^{2}}{2 E L_{D}(E)}, \\
& a_{3 T}=-\left(c_{0 T}+d_{0 T}\right), \\
& a_{2 T}=-E\left(c_{0 T}-d_{0 T}\right), \\
& a_{1 T}=-\left(E^{2}-2 \zeta^{2}\right)\left(c_{0 T}+d_{0 T}\right), \\
& a_{0 T}=1-E\left(E^{2}-2 \zeta^{2}\right)\left(c_{0 T}-d_{0 T}\right), \\
& L_{D}(E)=\left(E^{2}-E_{0}^{2}\right)^{2}+\Gamma^{2} E^{2} .
\end{aligned}
$$

Here we use the fully expanded form of the equations for $\epsilon_{1}(E)$, rather than the reduced version given by JM. ${ }^{10}$ With the expanded form, it is easier to describe the equations that result when $G(E)$ in Eqs. (1b) and (2b) is modified. For this purpose, we use a functional description of $I_{T L}(E)$ in terms of the photon energy dependent coefficients, written as: $I_{T L}\left(a_{i T} ; i=0,1,2,3 ; c_{0 T}, d_{0 T}\right)$ in Eq. (5).

\section{B. Determination of $I_{L}(E)$ assuming constant dipole matrix element}

As a modification of the Tauc theory, Cody proposed applying a constant dipole matrix element rather than a constant momentum matrix element, ${ }^{3}$ and the resulting expression $\left[\epsilon_{2}(E)\right]^{1 / 2} \propto[\alpha(E) n(E) / E]^{1 / 2} \propto\left(E-E_{g}\right)$ provides better fits to the absorption onset in $a$-Si:H. ${ }^{3,4,19,21}$ Thus, this modification yields a dielectric function just above the absorption onset of the form $\epsilon_{2}(E) \propto\left(E-E_{g}\right)^{2}$. However, because this expression diverges with increasing $E$, it cannot be applied directly as $G(E)$ in Eqs. (1b) and (2b). As a result an alternative empirical expression for $G(E)$ is

$$
G_{C}(E)=\frac{\left(E-E_{g}\right)^{2}}{\left(E-E_{g}\right)^{2}+E_{p}^{2}},
$$

Here $E_{p}$ defines a second transition energy (in addition to $E_{t}$ ) given by $E_{p}+E_{g}$, that separates the absorption onset behavior $\left[E<\left(E_{p}+E_{g}\right)\right]$ from the Lorentz oscillator behavior [E $>\left(E_{p}+E_{g}\right)$ ]. Specifically, if $E \approx E_{g}$ [so that $0<\left(E-E_{g}\right)$ $\left.\ll E_{p}\right]$, then $G(E) \rightarrow\left[\left(E-E_{g}\right) / E_{p}\right]^{2}$, whereas if $E \gg\left(E_{p}\right.$ $\left.+E_{g}\right)$ [so that $\left(E-E_{g}\right) \gg E_{p}$ ] then $G(E) \rightarrow 1$, as required to recover the desired Lorentz oscillator form for $\epsilon_{2}(E)$ at high energies. This second transition energy provides flexibility that is lacking in the Tauc-Lorentz expression. In fact, different functional forms for $G(E)$ can be applied to modify the oscillator function $L(E)$, depending on the desired shape of the absorption onset.

Using Eq. (18) in Eq. (2b) yields

$$
\begin{aligned}
I_{C L}\left(a_{i C} ; i=\right. & \left.0,1,2,3 ; b_{0 C}, b_{1 C}, c_{0 C}, d_{0 C}\right) \\
= & I_{T L}\left(a_{i C} ; i=0,1,2,3 ; c_{0 C}, d_{0 C}\right) \\
& +\frac{2 A E_{0} \Gamma}{\pi}\left[b_{1 C}\left\{E_{g} I_{0 C}-\ln \left[\left(E_{t}-E_{g}\right)^{2}+E_{p}^{2}\right]^{1 / 2}\right\}\right. \\
& \left.+b_{0 C} I_{0 C}\right],
\end{aligned}
$$

where

$$
I_{0 C}=\frac{1}{E_{p}}\left[\frac{\pi}{2}-\tan ^{-1}\left(\frac{E_{t}-E_{g}}{E_{p}}\right)\right],
$$

and

$$
\begin{aligned}
& c_{0 C}=\frac{E G_{C}(E)}{2 L_{D}(E)}, \\
& d_{0 C}=\frac{-E\left(E+E_{g}\right)^{2}}{2 L_{D}(E)\left[\left(E+E_{g}\right)^{2}+E_{p}^{2}\right]},
\end{aligned}
$$

$$
\begin{aligned}
b_{1 C}= & Y^{-4}\left[2 E_{g} K^{2} b_{0 C}-L_{D}(E)\left(c_{0 C}+d_{0 C}\right)\right], \\
a_{3 C}= & -\left(b_{1 C}+c_{0 C}+d_{0 C}\right), \\
a_{2 C}= & -\left[b_{0 C}+2 E_{g} b_{1 C}+E\left(c_{0 C}-d_{0 C}\right)\right], \\
a_{1 C}= & -\left[2 E_{g} b_{0 C}-\left(K^{2}-F^{2}\right) b_{1 C}+\left(E^{2}-2 \zeta^{2}\right)\right. \\
& \left.\times\left(c_{0 C}+d_{0 C}\right)\right], \\
a_{0 C}= & 1+\left(K^{2}-F^{2}\right) b_{0 C}+2 E_{g} K^{2} b_{1 C}-E\left(E^{2}-2 \zeta^{2}\right) \\
& \times\left(c_{0 C}-d_{0 C}\right), \\
F^{2}= & E_{p}^{2}+E_{g}^{2}, \\
K^{2}= & 2 F^{2}+2 \zeta^{2}-4 E_{g}^{2},
\end{aligned}
$$

$$
Y^{4}=E_{0}^{4}+F^{2}\left(K^{2}-F^{2}\right)-4 E_{g}^{2} K^{2} .
$$

In Eq. (19), $I_{T L}\left(a_{i C} ; i=0,1,2,3 ; c_{0 C}, d_{0 C}\right)$, is given by Eqs. (5)-(10) and (17), but with the energy dependent coefficients $\left\{a_{i T} ; i=0,1,2,3 ; c_{0 T}, d_{0 T}\right\}$ of Eqs. (11)-(16), replaced by the coefficients $\left\{a_{i C} ; i=0,1,2,3 ; c_{0 C}, d_{0 C}\right\}$ of Eqs. (21), (22), and (25)-(28).

\section{Determination of $I_{U}(E)$ assuming an exponential absorption tail}

As noted earlier, for hydrogenated group IV semiconductors used in devices, the Urbach tail in $\epsilon_{2}(E)$, defined by Eq. (1a), generates only a very small contribution to $\epsilon_{1}(E)$ through the Kramers-Kronig relations. By solving the inte- 



FIG. 1. Optical properties of 0.5-1.5- $\mu$ m-thick samples of (a) $a$ - $\mathrm{Si}: \mathrm{H}$, (b) $a-\mathrm{Si}_{1-x} \mathrm{Ge}_{x}: \mathrm{H}$, and (c) $a-\mathrm{Si}_{1-x} \mathrm{C}_{x}: \mathrm{H}$, expressed as $\left(\epsilon_{1}, \epsilon_{2}\right)$ (top panel) and ( $\left.n, \alpha\right)$ (bottom panel). These results were obtained at room temperature by combining dual beam photoconductivity spectroscopy, transmittance and reflectance spectroscopy, and spectroscopic ellipsometry measurements. The solid lines are fits to the data using the seven parameter expression of Eqs. (1)-(3), (5)-(10), and (17)-(32).

gral $I_{U}(E)$ in Eqs. (2), we can include this contribution for completeness and, in addition, provide the capability of simulating the optical functions for a material in which exponentially increasing absorption dominates the near-band edge behavior. In this latter situation, we can use the Tauc Law for $G(E)$, setting $E_{g}=0$. This eliminates $G_{T}(E)$ from the near-band edge behavior, allowing the Urbach tail to be connected directly to the Lorentz oscillator. In the most general case for $I_{U}(E)$ in Eqs. (2), integration yields

$$
\begin{aligned}
I_{U}(E)= & \frac{E_{1}}{\pi E}\left\{\exp \left(\frac{E-E_{t}}{E_{u}}\right)\left[E i\left(\frac{E_{t}-E}{E_{u}}\right)-E i\left(-\frac{E}{E_{u}}\right)\right]\right. \\
& \left.-\exp \left(\frac{-\left(E+E_{t}\right)}{E_{u}}\right)\left[E i\left(\frac{E_{t}+E}{E_{u}}\right)-E i\left(\frac{E}{E_{u}}\right)\right]\right\},
\end{aligned}
$$

where $E i(x)$ designates the exponential integral function defined by $\operatorname{Ei}(x)=\int_{-\infty}^{x}[\exp (t) / t] d t$. Algorithms for evaluation of this function can be found in reference texts (see, for example, Ref. 22).

As might be expected, the addition of the Urbach tail introduces difficulties in the evaluation of the KramersKronig integrals of Eqs. (2) for $E=0$ and $E_{t}$. First, $E i$ $\left( \pm E / E_{u}\right)$ in Eq. (32) diverges negatively as $E \rightarrow 0$. For small $x$, however, $E i(x) \approx \ln |x|+\gamma_{E}+x+\ldots$, where $\gamma_{E}$ $=0.57721566 \ldots$ is Euler's constant, ${ }^{22}$ and the two divergences for the positive and negative terms in Eq. (32) cancel, leading to an expression of the form $I_{U}(E) \rightarrow\left(2 E_{1} / \pi E_{u}\right)$ $\times\left[\exp \left(-E_{t} / E_{u}\right)\right]$ as $E \rightarrow 0$. Such behavior is of no practical consequence for $a-\mathrm{Si}: \mathrm{H}$ based materials since the contribution to $\epsilon_{1}(E)$ will be negligible owing to the $\exp \left(-E_{t} / E_{u}\right)$ 


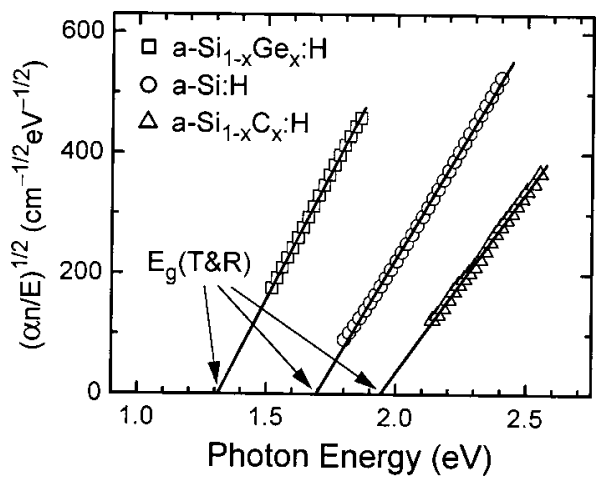

FIG. 2. Illustration of the procedure used to determine the optical gap value $E_{g}(\mathrm{~T} \& \mathrm{R})$ for $a-\mathrm{Si}: \mathrm{H}$ based alloys from transmittance and reflectance spectroscopy alone. This analysis assumes parabolic band densities of states, a constant dipole matrix element vs photon energy, and no states below the parabolic band edges.

factor. Second, $E i\left( \pm\left[E_{t}-E\right] / E_{u}\right)$ in Eq. (32) diverges as $E \rightarrow E_{t}$. For small values of $\left(E_{t}-E\right)$, however, the divergence in $\epsilon_{1}\left(E_{t}\right)$ from the term in $E i\left[\left(E_{t}-E\right) / E_{u}\right]$ is cancelled by the divergence that occurs in either $I_{T L}(E)$ or $I_{C L}(E)$ [see Eq. (5)] due to the term in $\ln \left|E-E_{t}\right|$. In this case, in order to evaluate $\epsilon_{1}$ at $E_{t}$, all terms (including their prefactors) in Eq. (2b) that diverge at $E=E_{t}$ can be replaced by the single term $\left\{G\left(E_{t}\right) L\left(E_{t}\right) / \pi\right\}\left\{\gamma_{E}-\ln E_{u}-E i\left(-E_{t} / E_{u}\right)\right\}$. As a result, a weak feature appears in $\epsilon_{1}(E)$ centered at $E$ $=E_{t}$ due to the discontinuity in the first derivative of $\epsilon_{2}(E)$ at $E_{t}$, denoted $\epsilon_{2}^{\prime}\left(E_{t}\right)$. Again for $a$-Si:H based alloys, the magnitude of $\epsilon_{2}\left(E_{t}\right)$ is small and the best fit parameters lead to near-continuity of $\epsilon_{2}^{\prime}\left(E_{t}\right)$. Thus, the resulting feature in $\epsilon_{1}(E)$ for $E$ near $E_{t}$ is below the sensitivity of the measurement techniques.

\section{RESULTS}

Figure 1 (points) shows typical experimental data for $0.5-1.5-\mu \mathrm{m}$-thick films of (a) $a-\mathrm{Si}: \mathrm{H}$, (b) $a-\mathrm{Si}_{1-x} \mathrm{Ge}_{x}: \mathrm{H}$, and (c) $a-\mathrm{Si}_{1-x} \mathrm{C}_{x}: \mathrm{H}$ deposited by PECVD on glass substrates. These experimental data are presented in terms of $\left(\epsilon_{1}, \epsilon_{2}\right)$ in the upper panels and $(n, \alpha)$ in the lower panels, and were obtained by combining three different methods. ${ }^{21}$ $\mathrm{SE}$ was performed over the strongly absorbing (opaque) regime, with typical ranges of $2.0-5 \mathrm{eV}$ for $a-\mathrm{Si}_{1-x} \mathrm{Ge}_{x}: \mathrm{H}$ and $2.5-5 \mathrm{eV}$ for $a-\mathrm{Si}_{1-x} \mathrm{C}_{x}: \mathrm{H}$. Transmission and reflection (T\&R) spectroscopy was performed over the intermediate photon energy regime of semitransparency, with typical ranges of $1.5-2.0 \mathrm{eV}$ for $a-\mathrm{Si}_{1-x} \mathrm{Ge}_{x}: \mathrm{H}$ and $2.0-2.5 \mathrm{eV}$ for $a-\mathrm{Si}_{1-x} \mathrm{C}_{x}: \mathrm{H}$. Dual beam photoconductivity (DBPC) was performed over the low energy Urbach tail regime of very low absorption, with typical ranges of $1.0-1.5 \mathrm{eV}$ for $a-\mathrm{Si}_{1-x} \mathrm{Ge}_{x}: \mathrm{H}$ and $1.5-2.0 \mathrm{eV}$ for $a-\mathrm{Si}_{1-x} \mathrm{C}_{x}: \mathrm{H}$. The effects of surface roughness and native oxide overlayers on the SE data in all cases were corrected by ensuring a match in $\epsilon_{2}$ or $\alpha$ in the region of overlap between the SE and T\&R spectra. Using this criterion and adopting a fixed contribution of $15 \AA$ for the native oxide thicknesses, the surface roughness thicknesses extracted from the SE data were found to be consistent with atomic force microscopy measurements, in view of previously established correlations. ${ }^{23}$
The samples in Fig. 1 are identified by their optical band gaps $E_{g}(\mathrm{~T} \& \mathrm{R})$ as deduced from extrapolations of the nearband gap optical functions using the constant dipole matrix element approach described earlier, according to $\left[\epsilon_{2}(E)\right]^{1 / 2}$ $\propto[\alpha(E) n(E) / E]^{1 / 2} \propto\left(E-E_{g}\right)$. Figure 2 depicts optical band gap plots for the films of Fig. 1, including data only from T\&R spectroscopy, which is the situation faced by most researchers. Identifying the samples by their band gaps is more useful from the standpoint of electronic device applications than identifying them by their alloy compositions. Owing to variations in $\mathrm{H}$ content, the alloy composition $x$ does not uniquely specify the optical band gap.

Under optimal circumstances, i.e., for the thicker films $(>1 \mu \mathrm{m})$, the data from SE, T\&R, and DBPC can be spliced together to obtain the complex dielectric function $\left(\epsilon_{1}, \epsilon_{2}\right)$ or the index of refraction and absorption coefficient $(n, \alpha)$ over the full range without any gaps. For the thinnest films $(\sim 0.5$ $\mu \mathrm{m})$, however, limitations exist, and two such limitations are illustrated in Fig. 1. First, Fig. 1(b) illustrates a $\sim 0.15 \mathrm{eV}$ gap between the T\&R and DBPC data. Since the DBPC measurement is a relative one, however, the normalization constant is found by comparing the DBPC data to an extrapolated result from Eq. (1a). In the extrapolation, $E_{u}$ is determined from the slope of the DBPC data; $E_{t}$ is obtained from the expression $E_{t}=E_{g}+2 E_{u}$ (see Sec. IV); and the parameters $E_{g}$ and $E_{1}$ are extracted in the best fit to the T\&R and SE data alone. This approach is similar to that described previously, ${ }^{24}$ and its validity has been supported by photothermal deflection spectroscopy, which is an alternative method for obtaining $\alpha$ over the Urbach tail. Second, Fig. 1(c) shows that data in $\epsilon_{1}$ and $n$ are unavailable in the intermediate and low energy ranges. First of all, $n$ cannot be obtained from DBPC measurements, and second it is often difficult to extract $n$ from T\&R unless the film is optically thick enough to exhibit a high density of interference fringes. In such cases as in Figs. 1(b) and 1(c), the interpolated or extrapolated fit shown as the solid lines in Fig. 1 (discussed further, later in this section) can still be used for simulation purposes due to the Kramers-Kronig consistency of the model. ${ }^{25}$

The fits to the full optical spectra such as those in Fig. 1 require as many as eight free parameters. Three parameters $\left\{A, E_{0}, \Gamma\right\}$ are associated with the Lorentz oscillator $L(E)$, two $\left\{E_{g}, E_{p}\right\}$ with the band edge function $G_{C}(E)$, two $\left\{E_{u}, E_{t}\right\}$ with the Urbach tail, and a final parameter $\epsilon_{1 \infty}$ with the constant contribution to $\epsilon_{1}(E)$. In such fitting, the number of free parameters can sometimes be reduced by exploiting (i) obvious functional relationships in the data, (ii) connections between free parameters, and (iii) parameters that are independent of the nature of the sample. Within this third category, for example, we can fix $\epsilon_{1 \infty}$ to unity. If this parameter is allowed to vary, values of unity within the range of the confidence limits are usually obtained, an improvement over the situation with the Tauc-Lorentz expression, for which near zero or negative values of $\epsilon_{1 \infty}$ are found for the $a-\mathrm{Si}_{1-x} \mathrm{Ge}_{x}: \mathrm{H}$ samples. As a result, all the fits of Fig. 1 shown as the solid lines utilize seven free parameters. Additional possible approaches to reduce the number of free parameters further are noted later. 


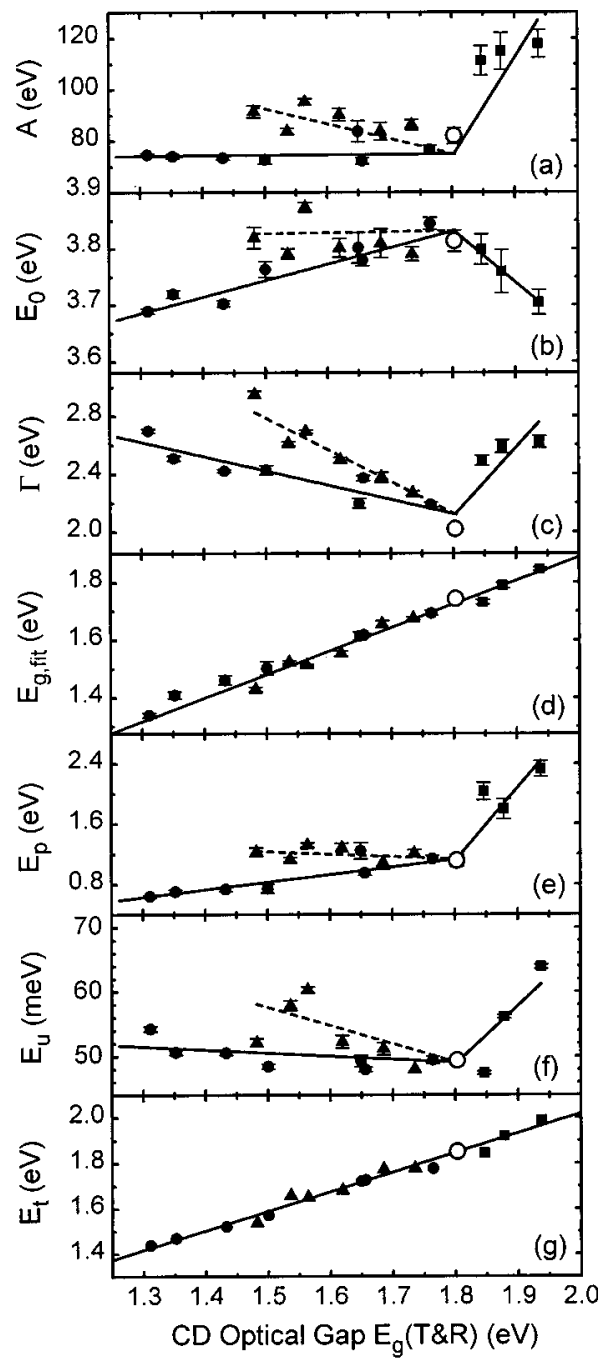

FIG. 3. The seven parameters in the analytical expression for the optical functions of $a-\mathrm{Si}: \mathrm{H}$ based alloy thin films, plotted as a function of the optical gap $E_{g}(\mathrm{~T} \& \mathrm{R})$. These include the Lorentz oscillator parameters (a) $A$, (b) $E_{0}$, and (c) $\Gamma$; the band edge parameters (d) $E_{g, \text { fit }}$ and (e) $E_{p}$; and the Urbach tail parameters (f) $E_{u}$ and (g) $E_{t}$. Results from three different sample sets are included: optimized (solid circles) and nonoptimized (solid triangles) $\quad a-\mathrm{Si}_{1-x} \mathrm{Ge}_{x}: \mathrm{H}, \quad$ both with $E_{g}(\mathrm{~T} \& \mathrm{R}) \leqslant 1.803 \mathrm{eV}$, and $a-\mathrm{Si}_{1-x} \mathrm{C}_{x}: \mathrm{H}$ (solid squares) with $E_{g}(\mathrm{~T} \& \mathrm{R}) \geqslant 1.803 \mathrm{eV}$. The lines indicate fits to the results for the sample sets - optimum $a$-Si:H with $E_{g}(\mathrm{~T} \& \mathrm{R})$ $=1.803 \mathrm{eV}$ (open circle) being included in each set. The broken line represents the nonoptimized $a-\mathrm{Si}_{1-x} \mathrm{Ge}_{x}: \mathrm{H}$ sample set. The intercepts for the nonoptimized $a-\mathrm{Si}_{1-x} \mathrm{Ge}_{x}: \mathrm{H}$ and the $a-\mathrm{Si}_{1-x} \mathrm{C}_{x}: \mathrm{H}$ sample sets are forced to match that of the optimized $a-\mathrm{Si}_{1-x} \mathrm{Ge}_{x}: \mathrm{H}$ set at the gap energy $E_{g}(\mathrm{~T} \& \mathrm{R})=1.803 \mathrm{eV}$.

In the least-squares regression analysis procedure applied to obtain the fits in Fig. 1, $\left(\epsilon_{1}, \epsilon_{2}\right)$ are used to represent the SE data, and $\left(\epsilon_{1}, \log \epsilon_{2}\right)$ are used to represent the T\&R and DBPC data. The dominant error in the determination of $\left(\epsilon_{1}, \epsilon_{2}\right)$ by $\mathrm{SE}$ arises not from the actual measurement, but rather from confidence limits of $\sim \pm 1 \AA$ on the surface roughness layer thickness assigned in data reduction. Because it is necessary to correct for the surface roughness layer analytically in order to extract the true optical functions, this thickness error translates into an error in $\left(\epsilon_{1}, \epsilon_{2}\right)$. In contrast, the dominant error in the determination of $\left(\epsilon_{1}, \log \epsilon_{2}\right)$ by $\mathrm{T} \& \mathrm{R}$ spectroscopy arises from irreproduc- ibilities of $\sim \pm 0.0025$ inherent in the transmittance and reflectance measurements. The errors in $\left(\epsilon_{1}, \epsilon_{2}\right)$ are incorporated into assessments of the suitability of the fits in Fig. 1 via $\chi^{2}$, the biased estimator of the mean square deviation [not to be confused with $\chi$ in Eq. (10)]. In fact, for the fits of Fig. 1 values of $\chi^{2}$ within the range of $0.5-2$ are found. ${ }^{12}$ Such values near unity imply that the fits are acceptable considering the inherent limitations in measurement and data reduction. In contrast, the corresponding values of $\chi^{2}$ in fits using the constant momentum matrix element approach of Sec. II A (along with the constraint that $\epsilon_{1 \infty} \geqslant 1$ ) are higher by factors ranging from 1.5 to 6 (for $a-\mathrm{Si}_{1-x} \mathrm{C}_{x}: \mathrm{H}$ to $a-\mathrm{Si}_{1-x} \mathrm{Ge}_{x}: \mathrm{H}$, respectively). Improved fits for this latter approach are obtained if $\epsilon_{1 \infty}$ is allowed to assume unphysical values below unity; however, the $\chi^{2}$ values are still higher than those obtained for the corresponding constant dipole matrix element approach of Sec. II B. Thus, based on statistical considerations of the fits, our analytical model is adequate for the purposes of simulation and analysis of the optical properties of $a$-Si:H-based alloys, and it provides an improvement over previous models.

As noted earlier, an important goal of this work is to develop a database for the optical properties of $a$-Si:H based alloys that can be used in optical simulations of multilayer stacks such as multijunction solar cells. Figure 3 presents results that can be interpreted for such a purpose. Each data point within a given panel on this plot represents a different sample, identified by its optical gap, as determined from an extrapolation of the form $\epsilon_{2}^{1 / 2} \propto\left(E-E_{g}\right)$, assuming a constant dipole matrix element (see Fig. 2). This gap, denoted $E_{g}(\mathrm{~T} \& \mathrm{R})$, serves as the abscissa in Fig. 3. The reason for using this measurement of the optical band gap is its wide accessibility; it can be obtained from $T \& R$ spectra alone based on the assumption that $\epsilon_{1}$ is constant over the energy range of the measurement. ${ }^{21}$ The samples included in Fig. 3 range from the $a-\mathrm{Si}_{1-x} \mathrm{Ge}_{x}: \mathrm{H}$ film of Fig. 1(b) with the minimum $E_{g}(\mathrm{~T} \& \mathrm{R})=1.31 \mathrm{eV}$ to the $a-\mathrm{Si}_{1-x} \mathrm{C}_{x}: \mathrm{H}$ film of Fig. 1(c) with a maximum $E_{g}(\mathrm{~T} \& \mathrm{R})=1.94 \mathrm{eV}$. The seven panels of this figure describe $(\mathrm{a}, \mathrm{b}, \mathrm{c})$ the three Lorentz oscillator parameters $\left\{A, E_{0}, \Gamma\right\}$, (d,e) the two band edge parameters $\left\{E_{g, \text { fit }}, E_{p}\right\}$, and (f,g) the two Urbach tail parameters $\left\{E_{u}, E_{t}\right\}$, respectively, for the sample whose $E_{g}(\mathrm{~T} \& \mathrm{R})$ value is plotted along the abscissa. In Fig. 3, all seven parameters are obtained as the best fit to experimental data such as those in Figs. 1, with the exception of the Urbach tail slope $E_{u}$. In this plot, $E_{u}$ is deduced from an independent linear fit of the slope of the DBPC spectra. This approach yields narrower confidence limits and less scatter than the fit using the complete expression of Eqs. (1)-(3).

There are three sample sets in Fig. 3, and each set exhibits discernable trends in the seven parameters of the analytical expression for the optical functions.

(1) The first sample set includes optimum $a$-Si:H (open circle in Fig. 3) and a series of $a-\mathrm{Si}_{1-x} \mathrm{Ge}_{x}: \mathrm{H}$ alloys, the latter prepared by PECVD at $T=200-400^{\circ} \mathrm{C}$ from mixtures of $\mathrm{Si}_{2} \mathrm{H}_{6} / \mathrm{GeH}_{4} / \mathrm{H}_{2}$ (solid circles). In fact, all these samples are prepared with the maximum possible $\mathrm{H}_{2}$ dilution, but without crossing the thickness-dependent boundary into the mixed-phase amorphous+microcrystalline growth regime. 
TABLE I. Linear coefficients that connect the optical band gap $E_{g}(\mathrm{~T} \& \mathrm{R})$, as determined by $\mathrm{T} \& \mathrm{R}$ spectroscopy, and the seven photon energy independent parameters $P_{j}(j=1, \ldots, 7)$ in the analytical model for the optical functions. $B_{j}(j=1, \ldots, 7)$ are the intercepts of the relationships at $E_{g}(\mathrm{~T} \& \mathrm{R})=1.803 \mathrm{eV}$, and $C_{j k}(j=1, \ldots, 5 ; k$ $=1,2,3)$ and $C_{j}(j=6,7)$ are the slopes. For five of the seven parameters, three linear relationships are required, each having different slopes (as indicated by the subscript $k$ ) but the same intercept. These relationships are appropriate for the optimized $(k=1)$ and nonoptimized $(k=2) a-\mathrm{Si}_{1-x} \mathrm{Ge}_{x}: \mathrm{H}$ and for the $a-\mathrm{Si}_{1-x} \mathrm{C}_{x}: \mathrm{H}(k$ $=3$ ) sample sets. For the remaining two parameters only a single relationship is required for all three sample sets.

\begin{tabular}{|c|c|c|c|c|c|}
\hline \multirow{3}{*}{$\begin{array}{l}\text { Index } \\
j\end{array}$} & \multirow{3}{*}{$\begin{array}{c}\text { Parameter } \\
P_{j}\end{array}$} & \multicolumn{4}{|c|}{$P_{j}=B_{j}+C_{j k}\left[E_{g}(\mathrm{~T} \& \mathrm{R})-1.803 \mathrm{eV}\right]$} \\
\hline & & & $\begin{array}{c}E_{g}(\mathrm{~T} \& \mathrm{R}) \leqslant 1.8 \mathrm{eV} \\
\text { optimum } \\
a-\mathrm{Si}_{1-x} \mathrm{Ge}_{x}: \mathrm{H} \\
\quad(k=1)\end{array}$ & $\begin{array}{c}E_{g}(\mathrm{~T} \& \mathrm{R}) \leqslant 1.8 \mathrm{eV} \\
\text { nonoptimum } \\
a-\mathrm{Si}_{1-x} \mathrm{Ge}_{x}: \mathrm{H} \\
\quad(k=2)\end{array}$ & $\begin{array}{c}E_{g}(\mathrm{~T} \& \mathrm{R})>1.8 \mathrm{eV} \\
a-\mathrm{Si}_{1-x} \mathrm{C}_{x}: \mathrm{H} \\
\quad(k=3)\end{array}$ \\
\hline & & $B_{j}$ & $C_{j 1}$ & $C_{j 2}$ & $C_{j 3}$ \\
\hline 1 & $A(\mathrm{eV})$ & 74.94 & 1.505 & -60.70 & 387.3 \\
\hline 2 & $E_{0}(\mathrm{eV})$ & 3.832 & 0.2914 & 0.0155 & -0.9354 \\
\hline 3 & $\Gamma(\mathrm{eV})$ & 2.122 & -0.9931 & -2.197 & 4.737 \\
\hline 4 & $E_{p}(\mathrm{eV})$ & 1.134 & 1.001 & -0.3157 & 9.731 \\
\hline \multirow[t]{3}{*}{5} & $E_{u}(\mathrm{meV})$ & 49.03 & -4.866 & -28.31 & 90.63 \\
\hline & & \multicolumn{2}{|c|}{$P_{j}=B_{j}+C_{j}\left[E_{g}(\mathrm{~T} \& \mathrm{R})-1.803 \mathrm{eV}\right]$} & & \\
\hline & & $B_{j}$ & $C_{j}$ & & \\
\hline 6 & $E_{g, \text { fit }}(\mathrm{eV})$ & 1.727 & 0.8153 & & \\
\hline 7 & $E_{t}(\mathrm{eV})$ & 1.850 & 0.8601 & & \\
\hline
\end{tabular}

Such conditions lead to optimum electronic quality material for solar cells, which requires a combination of factors including good electron and hole transport properties and high stability against light-induced degradation. ${ }^{26}$

(2) The second sample set consists of the same optimum $a$-Si:H sample (open circle in Fig. 3), but other lower gap samples as well (triangles). These include $a-\mathrm{Si}: \mathrm{H}$ films prepared without $\mathrm{H}_{2}$ dilution, along with $a-\mathrm{Si}_{1-x} \mathrm{Ge}_{x}: \mathrm{H}$ alloy films prepared from mixtures of $\mathrm{SiH}_{4} / \mathrm{GeH}_{4} / \mathrm{H}_{2}$ or $\mathrm{Si}_{2} \mathrm{H}_{6} / \mathrm{GeH}_{4} / \mathrm{H}_{2}$ in which the maximal $\mathrm{H}_{2}$-dilution condition has not been reached for the successive increases in the flow of $\mathrm{GeH}_{4}$. Thus, these nonoptimum materials are expected to yield lower performance solar cells.

(3) The third sample set is more limited in scope and includes the optimum $a$-Si:H (open circle in Fig. 3) and $a-\mathrm{Si}_{1-x} \mathrm{C}_{x}: \mathrm{H}$ alloys prepared from $\mathrm{SiH}_{4} / \mathrm{CH}_{4} / \mathrm{H}_{2}$ under maximal $\mathrm{H}_{2}$-dilution conditions for highest performance solar cells (squares).

As shown in Fig. 3, the trends with $E_{g}(\mathrm{~T} \& \mathrm{R})$ in the parameters have been fit to three sets of linear relationships, enumerated to match the sample set. (1) One set of relationships spans the range from 1.3 to $1.8 \mathrm{eV}$, covering optimum $a$-Si:H and its optimized alloys with Ge (solid lines); (2) the second set of relationships spans the range from 1.5 to 1.8 $\mathrm{eV}$ and is appropriate for the narrower gap $a-\mathrm{Si}: \mathrm{H}$ and nonoptimized alloys with Ge (broken lines); and (3) the third set spans the range from 1.8 to $1.95 \mathrm{eV}$, covering the alloys with $\mathrm{C}$ (solid lines). For simplicity, the second and third sets of relationships are constrained to exhibit the same intercepts as the first relationships at the extrapolated gap value of $E_{g}(\mathrm{~T} \& \mathrm{R})=1.803 \mathrm{eV}$, corresponding to the abscissa value for optimum $a-\mathrm{Si}: \mathrm{H}$. In most cases, the best fit unconstrained intercepts are not far from these values. The reason for the constrained intercept approach will be clarified shortly. Among the seven parameters in Fig. 3, however, two exhibit a common linear relationship for all three sample sets. These unique parameters include $E_{g \text {,fit }}$, the best fit optical band gap from the full expressions of Eqs. (1)-(3), and $E_{t}$, the transition energy between the Urbach tail and the band edge region defined in Eq. (1a).

Table I presents the three functional relationships for the five parameters $\left\{P_{j} ; j=1, \ldots, 5\right\}=\left\{A, E_{0}, \Gamma, E_{p}, E_{u}\right\}$ and the single relationships for the two parameters $\left\{P_{j} ; j=6,7\right\}$ $=\left\{E_{g, \text { fit }}, E_{t}\right\}$. For the $j$ th parameter $P_{j}(j=1, \ldots, 7)$, the linear relationships in $E_{g}(\mathrm{~T} \& \mathrm{R})$ are given in one of two forms

$$
\begin{aligned}
P_{j}\left[E_{g}(\mathrm{~T} \& \mathrm{R})\right] & =B_{j}+C_{j k}\left[E_{g}(\mathrm{~T} \& \mathrm{R})-1.803 \mathrm{eV}\right] ; \\
j & =1, \ldots, 5 ; \quad k=1,2,3 ; \\
P_{j}\left[E_{g}(\mathrm{~T} \& \mathrm{R})\right] & =B_{j}+C_{j}\left[E_{g}(\mathrm{~T} \& \mathrm{R})-1.803 \mathrm{eV}\right] ; \\
j & =6,7 .
\end{aligned}
$$

For $j=1, \ldots, 5$, the sample set is designated by the index $k$ $=1,2,3$; for $j=6,7$, all sample sets give the same relationships, so the subscript $k$ is not needed. With the constrained intercept approach, $B_{j}$ for each $j$ is independent of the sample set index $k$. It is important to emphasize that from 19 linear coefficients of Table I, written as $\left\{\left(B_{j}, C_{j k}\right), j\right.$ $=1, \ldots, 5 ; k=1,3\}$ and $\left\{\left(B_{j}, C_{j}\right), j=6,7\right\}$, along with the value of $E_{g}(\mathrm{~T} \& \mathrm{R})=1.803 \mathrm{eV}$ (i.e., 20 parameters in total), one can calculate $\left(\epsilon_{1}, \epsilon_{2}\right)$ or $(n, \alpha)$ for an optimum quality alloy of any specified optical band gap, the latter obtained solely from T\&R spectra. One can also simulate the effect of a lower electronic quality on the optical functions of $a-\mathrm{Si}_{1-x} \mathrm{Ge}_{x}: \mathrm{H}$ by adjusting the slopes of the first relationships; i.e., by replacing the five parameters $\left\{C_{j k}, j\right.$ 
(a)

\begin{tabular}{|c|c|}
\hline glass & \\
\hline $\mathrm{SnO}_{2}$ & $500 \mathrm{~nm}$ \\
\hline p-layer & $10 \mathrm{~nm}$ \\
\hline i-layer, $E_{g}(T \& R)=1.6 \mathrm{eV}$ & $400 \mathrm{~nm}$ \\
\hline n-layer & $20 \mathrm{~nm}$ \\
\hline Zno & $180 \mathrm{~nm}$ \\
\hline Silver & $100 \mathrm{~nm}$ \\
\hline
\end{tabular}

(b)

\begin{tabular}{|c|c|}
\hline glass & \\
\hline $\mathrm{SnO}_{2}$ & $500 \mathrm{~nm}$ \\
\hline p-layer & $10 \mathrm{~nm}$ \\
\hline i-layer, $E_{g}(T \& R)=1.8 \mathrm{eV}$ & $93 \mathrm{~nm}$ \\
\hline n-layer & $10 \mathrm{~nm}$ \\
\hline p-layer & $10 \mathrm{~nm}$ \\
\hline i-layer, $E_{g}(T \& R)=1.6 \mathrm{eV}$ & $230 \mathrm{~nm}$ \\
\hline n-layer & $10 \mathrm{~nm}$ \\
\hline p-layer & \\
\hline i-layer, $E_{g}(T \& R)=1.4 \mathrm{eV}$ & $190 \mathrm{~nm}$ \\
\hline n-layer & $10 \mathrm{~nm}$ \\
\hline $\mathrm{ZnO}$ & $180 \mathrm{~nm}$ \\
\hline Silver & $100 \mathrm{~nm}$ \\
\hline
\end{tabular}

FIG. 4. Schematic multilayer structure and thicknesses for (a) an ideal single-junction $a$-Si:H $p-i-n$ solar cell, and (b) an ideal triple-junction $a-\mathrm{Si}: \mathrm{H}$ based $p-i-n$ solar cell. The optical functions of the intrinsic layers $(i$ layers) are shown in Fig. 5 as calculated from the analytical model, and the optical analysis of the devices is shown in Figs. 6 and 7. The thicknesses of the layers in (b) are extracted from the condition of current matching under AM 1.5 illumination.

$=1, \ldots, 5 ; k=1\}$ with $\left\{C_{j k}, j=1, \ldots, 5 ; k=2\right\}$. Without the constrained intercepts, an additional five parameters are needed in each case. It should be emphasized that the assignment of a "lower electronic quality" for sample set (2) is somewhat arbitrary; as a result, the data points exhibit considerably more scatter about the linear relationship $k=2$. This difficulty will be addressed in greater detail in Sec. V.

\section{DISCUSSION}

In addition to providing an effective algorithm for calculating the optical properties of amorphous semiconductors from a single specification of the optical gap, the parameter variations in Fig. 3 also provide insights into the effects of $\mathrm{Ge}$ and $\mathrm{C}$ alloying. In this section, the discussion will focus on the underlying origins of the linear trends in Fig. 3.

The parameters that can be given as single linear relationships for all samples will be considered first, starting with $E_{g, \text { fit }}$ in Fig. 3(d). Ideally, the best-fit and extrapolated (T\&R) optical gaps, distinguished in Fig. 3 as $E_{g \text {,fit }}$ and $E_{g}(\mathrm{~T} \& \mathrm{R})$, respectively, should be identical; however, Table I shows that $E_{g, \text { fit }}=(1.727 \mathrm{eV})+0.8153\left[E_{g}(\mathrm{~T} \& \mathrm{R})\right.$ $-1.803 \mathrm{eV}]$. The difference between $E_{g \text {,fit }}$ and $E_{g}(\mathrm{~T} \& \mathrm{R})$ [which is $0.076 \mathrm{eV}$ at $E_{g}(\mathrm{~T} \& \mathrm{R})=1.803 \mathrm{eV}$ and increases as a function of $\left.E_{g}(\mathrm{~T} \& \mathrm{R})\right]$ arises because the expression used to obtain $E_{g \text {,fit }}$ also includes the variation of the Lorentz oscillator function $L(E)$ with energy, and this leads to the systematic variations between the two gap values. In addition, fluctuations occur owing to the difficulty of fitting the full spectral range simultaneously with a relatively small number of free parameters (i.e., 7 - only 3 larger than that necessary to fit two linear functions).

The linearity of $E_{t}$ versus the extrapolated (T\&R) gap in Fig. $3(\mathrm{~g})$ is also of interest and reflects the requirement that the first derivative of $\epsilon_{2}(E)$ be continuous from the Urbach tail to the band edge region. If we neglect the photon energy dependence of $L(E)$ in this transition region, as well as terms in $E_{u}^{2}$ compared to $E^{2}$ and $E_{g}^{2}$, then the condition for

continuity of the derivative of $\epsilon_{2}(E)$ becomes $E_{t}=E_{g}$ $+2 E_{u}$. The gap in this case is the fitted value $E_{g \text {,fit }}$, and so a relationship of the form $E_{t} \approx E_{g \text {,fit }}+0.1 \mathrm{eV}$ is expected. In fact, a conversion of the coefficients in Table I yields the best-fit relationship $E_{t}=1.05 E_{g \text {,fit }}+0.028 \mathrm{eV}$, so that at $E_{g, \text { fit }}=1.30 \mathrm{eV}, E_{t}=1.39 \mathrm{eV}$, and at $E_{g, \text { fit }}=1.95 \mathrm{eV}, E_{t}$ $=2.08 \mathrm{eV}$. As a result of this relationship, $E_{t}$ can be fixed at $E_{t}=E_{g, \text { fit }}+2 E_{u}$ in the simulations. Thus, the total number of parameters required to fit data such as in Fig. 1 can be reduced to 6, and the number of independent linear coefficients required to generate the optical properties for arbitrary $E_{g}(\mathrm{~T} \& \mathrm{R})$ can be reduced from 20 to 18 . It should be noted that the relationship $E_{t}=E_{g}+2 E_{u}$ is also expected to hold if $G_{T}(E)$ from the Tauc law is used instead of $G_{C}(E)$.

Another important effect is the increase in Lorentz oscillator broadening parameter in Fig. 3(c) with alloying for all three sample sets. The broadening parameter is expected to scale inversely with the lifetime of carriers excited into states deep within the conduction and valence bands. ${ }^{18,19}$ The trends in $\Gamma$ in Fig. 3(c) are similar to those in $E_{u}$ in Fig. 3(f) since both are likely to be influenced by the enhanced bond length, bond angle, and chemical disorder that occurs upon alloying. However, additions of Ge exert a larger detrimental effect on $\Gamma$ in comparison with $E_{u}$; this is not unexpected considering that $\Gamma$ reflects extended valence and conduction band states, whereas $E_{u}$ reflects the localized state distribution in the valence band tail. ${ }^{20}$ On the basis of the results in Fig. 3(c), one can conclude that $\Gamma$ as measured by SE provides a sensitive method for the assessment of the electronic quality of $a-\mathrm{Si}_{1-x} \mathrm{Ge}_{x}: \mathrm{H}$ alloys. Figure 3(c) also shows that under optimum conditions the incorporation of small amounts of $\mathrm{C}$ is much more detrimental to the ordering than small amounts of Ge. For this reason, triple junction solar cells generally forgo the topmost $a-\mathrm{Si}_{1-x} \mathrm{C}_{x}: \mathrm{H}$ alloy layer, replacing it with a wider band gap $a-\mathrm{Si}: \mathrm{H}$ layer. ${ }^{27}$ The most highly ordered material from the standpoint of $\Gamma$ is $a-\mathrm{Si}: \mathrm{H}$ with an extrapolated gap of $1.80 \mathrm{eV}$, prepared by rf PECVD using a $\mathrm{H}_{2} / \mathrm{SiH}_{4}$ gas flow ratio of 10 . This so called "protocrystalline" material is obtained at the highest $\mathrm{H}_{2}$-dilution ratio possible without entering the microcrystalline regime. ${ }^{28}$

The other trends in Fig. 3 are less informative and in some cases exhibit correlations that mask the underlying origins. First considering $E_{p}$, one might expect this parameter to increase with increasing optical band gap, given its role as a transition energy between the band edge and Lorentz oscillator regimes. In fact, such a trend is observed for the optimum samples; for the full set of samples, however, it appears that $E_{p}$ also increases when the width of the Lorentz oscillator $\Gamma$ increases. Second, one might expect the oscillator resonance energy $E_{0}$ to increase with the optical gap, as well. The best fit variations are relatively small, however, within $\pm 0.1 \mathrm{eV}$, considering that the optical gap varies by $0.65 \mathrm{eV}$ over the full range of Fig. 3. The opposite trend, i.e., the decrease in $E_{0}$ with $E_{g}(\mathrm{~T} \& \mathrm{R})$, for the $a-\mathrm{Si}_{1-x} \mathrm{C}_{x}: \mathrm{H}$ alloys may be an effect of the large $E_{p}$ values which have a tendency to shift the peaks in the best fit simulated $\epsilon_{2}$ spectra to higher energies. As a result $E_{0}$ decreases to compensate for this effect and match the relatively weak shift to higher energy observed in the data [compare Figs. 1(a) and 1(c)]. Fi- 

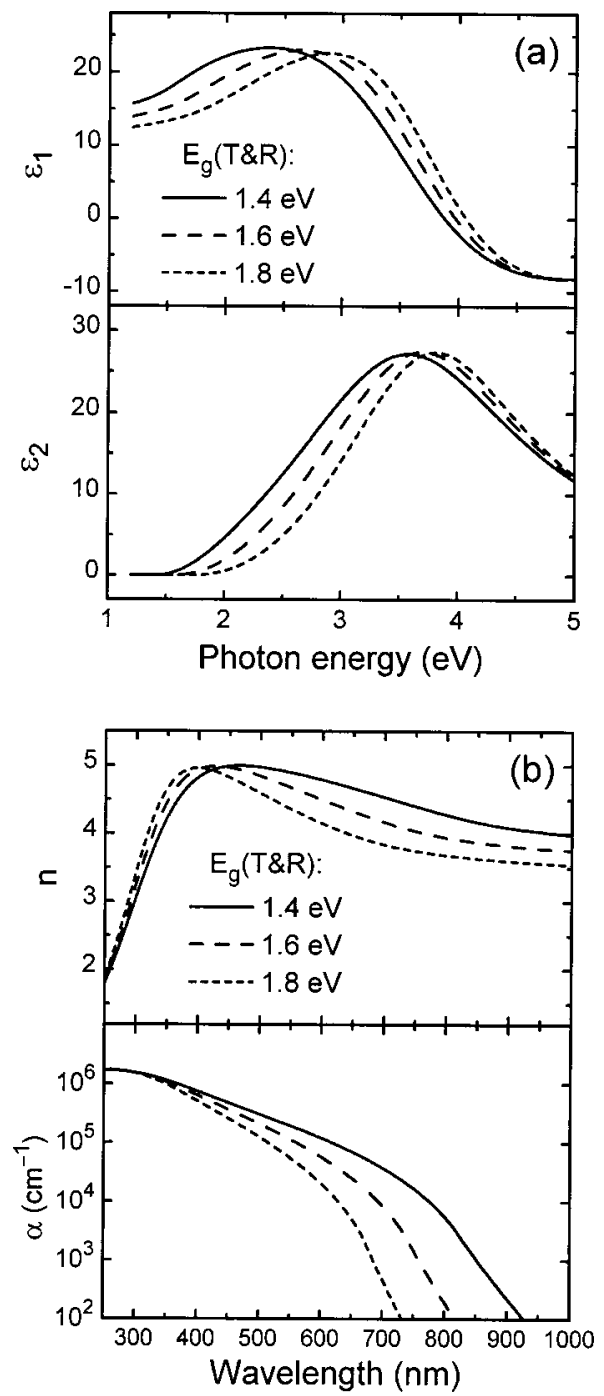

FIG. 5. Optical functions including (a) the real and imaginary parts of the dielectric functions $\left(\epsilon_{1}, \epsilon_{2}\right)$ and (b) the index of refraction and absorption coefficient $(n, \alpha)$ for optimum $a-\mathrm{Si}: \mathrm{H}$ based alloys of prespecified optical gaps. These results were computed from the seven parameter analytical model of Fig. 3 and the 15 parameters from Table I that describe sample set (1).

nally, because the amplitude $A$ of the Lorentz oscillator appears in the numerator of $\epsilon_{2}$ in Eq. (1b), whereas $E_{p}$ enters into the denominator, then there is a tendency for these parameters to be positively correlated. In fact, this behavior explains the relatively large values of $A$ for the $a-\mathrm{Si}_{1-x} \mathrm{C}_{x}: \mathrm{H}$ alloys. Without such a variation in $E_{p}, A$ would be nearly constant or even slightly decreasing with $E_{g}(\mathrm{~T} \& \mathrm{R})$ for all samples.

\section{SIMULATIONS AND APPLICATIONS}

Figures 4(a) and 4(b) depict two multilayer stacks, one corresponding to a single-junction $a-\mathrm{Si}: \mathrm{H} p-i-n$ solar cell and the other to a triple-junction $a-\mathrm{Si}: \mathrm{H}$ alloy $p-i-n$ solar cell. In both cases, the light enters the cell through the top glass "superstrate." Since the electronic performances of the intrinsic absorber (or active) layers of such devices appear to be approaching their ultimate limits, researchers are considering other avenues for increasing the conversion efficiency
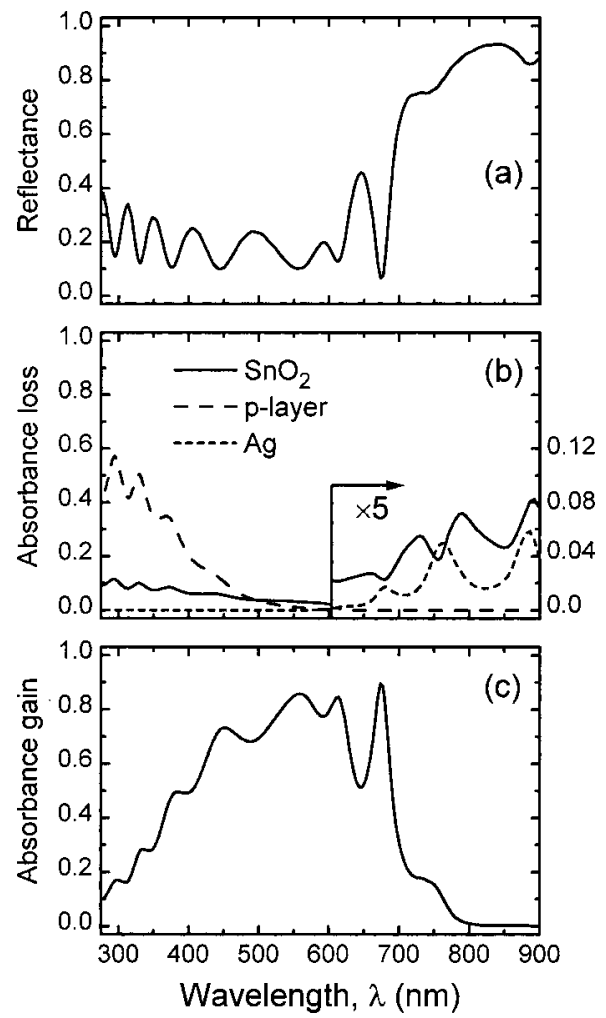

FIG. 6. Results of optical modeling for the single-junction $a$-Si:H based solar cell of Fig. 4(a), assuming an optimum $i$ layer $400 \mathrm{~nm}$ thick with 1.6 eV optical band gap (from T\&R spectroscopy). Computations include (a) the overall reflectance loss; (b) the absorbance losses in the $\mathrm{SnO}_{2}$, p-type $a-\mathrm{Si}: \mathrm{H}$, and Ag layers; and (c) the absorbance gain due to electron-hole pair creation in the intrinsic layer.

of solar cells. ${ }^{2}$ Thus, a subject of recent intense interest is the optical engineering of the devices for increased absorbance in the active layers. Two primary concepts have been implemented so far for such "light trapping" purposes. First, the $\mathrm{SnO}_{2}$ applied as a transparent conductor on the glass superstrate is textured to induce macroscopic roughness at the successive interfaces of the multilayer. As a result, light is scattered at these interfaces, increasing the average optical path length as well as the effective absorbance for light waves within the active layers. Second, a $\mathrm{ZnO} / \mathrm{Ag}$ or $\mathrm{ZnO} / \mathrm{Al}$ retroreflector is incorporated at the back surface of the device. This structure reflects red and near-infrared light waves back through the device in a second pass, and likewise enhances the effective absorbance of the active layers, particularly when used in conjunction with macroscopic roughness at the back $\mathrm{ZnO} /$ metal interface.

Accurate multilayer optical simulation is required to assess the efficacy of light trapping schemes, as well as to develop advanced methods for efficiency enhancement based on optical engineering. Different simulation approaches have been developed and applied, including simple models that incorporate incoherent summation of partial waves, as well as more complicated models that incorporate either coherent or incoherent summation, depending on the layer thickness. ${ }^{29,30}$ Irrespective of the approach, all such models require as a foundation the optical functions of the component layers of the solar cell. In assigning the optical functions 

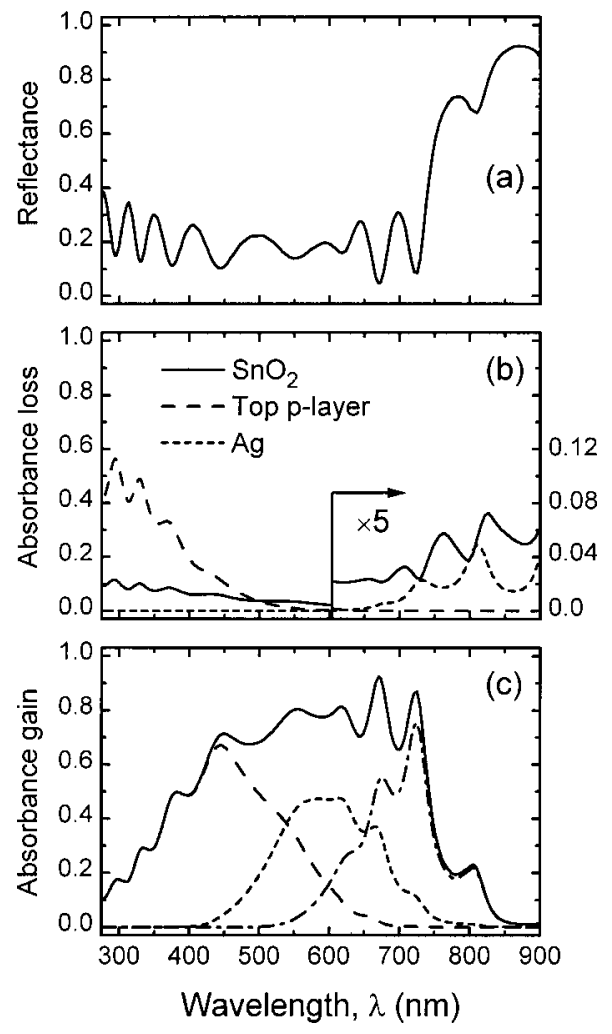

FIG. 7. Results of optical modeling for the triple-junction $a$-Si:H based solar cell of Fig. 4(b), assuming intrinsic layer optical band gaps of 1.8, 1.6, and $1.4 \mathrm{eV}$ (from T\&R spectroscopy) and thicknesses that yield currentmatched operation $\left(6.6 \mathrm{~mA} / \mathrm{cm}^{2}\right.$ under global AM 1.5). Computations include (a) the overall reflectance loss; (b) the absorbance losses in the $\mathrm{SnO}_{2}$, $p$-type $a$ - $\mathrm{Si}: \mathrm{H}$, and Ag layers; and (c) the absorbance gain due to electronhole pair creation in the three intrinsic layers. The latter spectrum is split into three parts corresponding to absorption in the $1.8 \mathrm{eV}$ (left), $1.6 \mathrm{eV}$ (center), and $1.4 \mathrm{eV}$ (right) intrinsic layer materials.

for the structures of Fig. 4, however, one is faced with two problems. First, the optical functions of the component layers depend on the fabrication method; and second, the optical functions of the active layers are required for any assigned value of the optical gap $E_{g}(\mathrm{~T} \& \mathrm{R})$ from $\sim 1$ to $2 \mathrm{eV}$. The second problem must be solved in order to apply optical modeling to optimize the optical gaps and thicknesses of the layers for spectral splitting and current matching purposes in multijunction devices. Previous research on optical modeling has yet to address problems of this nature. In this work, we have solved both such problems for the most important materials of the device, the intrinsic absorber layers.

As a demonstration, Figs. 5(a) and 5(b) show $\left(\epsilon_{1}, \epsilon_{2}\right)$ versus photon energy $E$, as well as $(n, \alpha)$ versus wavelength, respectively, for hypothetical optimum quality $a$-Si:H based alloys of prespecified optical gaps of $E_{g}(\mathrm{~T} \& \mathrm{R})=1.4,1.6$, and $1.8 \mathrm{eV}$. These results are calculated from the analytical expressions given in Eqs. (1)-(3), (5)-(10), and (17)-(32), employing the coefficients in Table I and the optical band gap energy at which the common intercepts occur $\left[E_{g}(\mathrm{~T} \& \mathrm{R})=1.803 \mathrm{eV}\right]$. The minimum number of coefficients required to set up a database over this range of $E_{g}(\mathrm{~T} \& \mathrm{R})$ is 13 , reduced from 20 by assuming (i) that $E_{t}$ is defined by $\left(E_{g, \text { fit }}, E_{u}\right)$ and (ii) that one is not interested in employing the $a-\mathrm{Si}_{1-x} \mathrm{C}_{x}: \mathrm{H}$ alloy materials in the multijunc- tion solar cell. This number is remarkably small, considering the relatively complex optical structure in Fig. 5.

The results of Fig. 3 and Table I suggest a simple procedure for incorporating the deposition dependence in the modeling. Because sample set (2) lacks uniqueness, however, this procedure requires additional measurements of the Urbach absorption tail for any alternative series of samples to be used as the basis for optical function simulation [denoted sample set $(n)]$. This procedure can be implemented as follows. If sample set $(n)$ includes optimized $a-\mathrm{Si}: \mathrm{H}$ with a $1.8 \mathrm{eV}$ gap (e.g., by preparation at $200^{\circ} \mathrm{C}$ under maximal $\mathrm{H}_{2}$-dilution conditions), but nonoptimized $a$ - $\mathrm{Si}_{1-x} \mathrm{Ge}_{x}: \mathrm{H}$ alloys, then one can adjust the slopes of the linear variations in Table I as directed by sample set (2). First, the variation of the energy $E_{u}$ with optical gap $E_{g}(\mathrm{~T} \& \mathrm{R})$ can be determined for sample set $(n)$. The slope of this relationship denoted $C_{5 n}$ will be less (i.e., more negative) than $C_{51}=-5 \mathrm{meV} / \mathrm{eV}$, the value for sample set (1) and possibly as small as (or even smaller than) $C_{52}=-28 \mathrm{meV} / \mathrm{eV}$, the value for sample set (2) [see Eq. (33) and Table I], whereas the intercept at $E_{g}(\mathrm{~T} \& \mathrm{R})=1.803 \mathrm{eV}$ can be constrained to $B_{5} \sim 49 \mathrm{meV}$. Then the other slopes $C_{j n}(j=1, \ldots, 4)$, associated with the four parameters $\left\{A, E_{0}, \Gamma, E_{p}\right\}$, respectively, can be established from linear interpolations of the coefficients in Table I versus sample quality according to

$$
C_{j n}=\left(C_{j 2}-C_{j 1}\right)\left(\frac{C_{5 n}-C_{51}}{C_{52}-C_{51}}\right)+C_{j 1} .
$$

The other two parameters $\left\{E_{g, \text { fit }}, E_{t}\right\}$ do not depend sensitively on the sample quality as is evident from Fig. 3, and so no adjustment of the slopes is needed for these. The resulting coefficient set $\left\{\left(B_{j}, C_{j n}\right), j=1, \ldots, 5 ;\left(B_{j}, C_{j}\right), j=6,7\right\}$ is then appropriate for establishing the optical function database relevant for sample set $(n)$. Alternative approaches for establishing the appropriate set of linear coefficients are certainly possible, depending on the nature of the sample set. With the approach just described the Urbach tail slope is a secondary parameter, in addition to the optical gap value $E_{g}(\mathrm{~T} \& \mathrm{R})$, that can be applied to establish the relevant optical function database for any set of alloys. A larger sample set (2) is needed, however, to place this procedure on a stronger foundation.

Next we provide examples of the multilayer optical modeling enabled by the set of optical functions developed here for the highest quality $a$-Si:H alloys. Figures 6 and 7 represent results obtained for the structures of Figs. 4(a) and 4(b), respectively, adopting the thicknesses listed there. In fact, the thicknesses in Fig. 4(b) are chosen for current matching in the three separate active layers of the device at a level of $6.6 \mathrm{~mA} / \mathrm{cm}^{2}$, assuming a global air mass 1.5 solar spectrum. In Figs. 6 and 7, we separate the optical processes into (a) reflectance losses, (b) parasitic absorbance losses by the inactive layers, the most important losses being those in the $\mathrm{SnO}_{2}$, the top-most $p$ layer, and the $\mathrm{Ag}$ retroreflector, and (c) absorbance gain by the active layer(s). For simplicity, the glass is assumed to be nonabsorbing throughout, and the $\mathrm{ZnO}$ is assumed to be nonabsorbing below its band gap. Thus, losses in these components are negligible. For the multijunction device, the absorbance gain is in turn divided into 
three parts, corresponding to absorption by the top, middle, and bottom cell active layers [left, center, and right curves, respectively, in Fig. 7(c)].

In the optical model, partial waves within the glass are assumed to be incoherent; complete coherence is retained elsewhere in the multilayer structure. Hence, a complex interference pattern is generated by multiple reflections between the boundaries of the thickest layers. Since no microscopic or macroscopic roughness is assumed in the model, this interference pattern is more pronounced than that observed in actual device structures.

The optical functions for the other component materials of the solar cell structures of Figs. 4 are obtained as described in detail elsewhere. ${ }^{6}$ These materials include the glass superstrate, the transparent conducting oxides - both doped $\mathrm{SnO}_{2}$ and $\mathrm{ZnO}$, the retroreflecting metal $\mathrm{Ag}$, and the contact layers of $p$ - and $n$-type $a$-Si:H. In fact, the optical functions of the contact $p$ layer were determined for a protocrystalline film incorporated in an optimized solar cell structure, as discussed in a recent publication. ${ }^{31}$ The same general strategy is being pursued to characterize the optical functions of all other solar cell materials as has been described above for the $a-\mathrm{Si}: \mathrm{H}$ based active layers. Specifically, the optical functions are to be developed as analytical functions that include photon energy independent parameters connected to basic properties of the material. In this way, the optical engineering of the device can proceed on the basis of a fundamental understanding of the component layers.

\section{CONCLUDING REMARKS}

In this effort, we have focused on the general goal of relating the complete optical functions for $a$ - $\mathrm{Si}: \mathrm{H}$ based alloys to one or two readily accessible optical parameters, in particular, the optical band gap and the Urbach tail slope as deduced from T\&R and photoconductivity spectroscopies, respectively. [By "optical functions" one means the photon energy (or wavelength) dependence of the real and imaginary parts of the dielectric function $\left(\epsilon_{1}, \epsilon_{2}\right)$ or the index of refraction and absorption coefficient $(n, \alpha)$.] The motivation of this effort is to develop a database of optical functions for use in modeling the performance of optoelectronic devices, such as solar cells and light detectors, as well as to fit optical measurements on complex device structures to extract not only layer thicknesses, but also basic material properties such as optical band gaps.

As a first step toward this goal, an analytical expression has been developed for the optical functions of $a$-Si:H based alloys. This expression provides advantages over previous ones devised specifically to analyze SE data in that it can be applied to fit optical functions combined from different methods, particularly those with higher sensitivity to $\alpha$ such as T\&R and photoconductivity spectroscopies, as well as SE. Thus, the range of $\alpha$ that can be fitted extends from $10 \mathrm{~cm}^{-1}$ in the below-band gap region to $>10^{6} \mathrm{~cm}^{-1}$ in the band-toband region. The analytical model for the imaginary part of the dielectric function $\epsilon_{2}=(\hbar c n \alpha) / E$ features an Urbach exponential absorption tail region, a band edge absorption region consistent with parabolic bands and a constant dipole matrix element, and a Lorentz oscillator region at high energies. At the minimum, only six energy independent free parameters are required in the analytical model, one to describe the Urbach tail, two to describe the band edge function, and three to describe the oscillator.

Studies of a set of optimum $a-\mathrm{Si}: \mathrm{H}$ and its alloys with $\mathrm{Ge}$ and $\mathrm{C}$ spanning the range of optical band gaps (as determined by an accessible method based on T\&R spectroscopy) from 1.30 to $1.95 \mathrm{eV}$, reveal clear trends as a function of the band gap. Such trends can be fit using two linear segments, one for $a-\mathrm{Si}_{1-x} \mathrm{Ge}_{x}: \mathrm{H}$ and the other for $a-\mathrm{Si}_{1-x} \mathrm{C}_{x}: \mathrm{H}$. From a minimum of 18 coefficients associated with these segments, the optical functions of an optimum alloy material of any specified optical band gap can be generated for the purposes of optical modeling. Approaches for simulating the optical properties of poorer quality materials can be incorporated as well by replacing 5 of the coefficients.

Limitations of the overall approach must be emphasized as well. The sample set characterized here consisted of films prepared by the widely studied, low-temperature $\left(\leqslant 400{ }^{\circ} \mathrm{C}\right)$ process of plasma-enhanced chemical vapor deposition under conditions yielding solar cell quality materials. The same polynomial coefficients are not expected to apply to materials prepared by different methods; however, the general approach can be repeated for such materials and an alternative set of coefficients established. Finally, it may be argued that the free parameters in the analytical expression for the optical functions should be related, not to the optical parameters themselves, but rather to the alloy composition, $\mathrm{H}$ content and bonding, and void fraction (see, for example, Ref. 32). We use the optical parameters because they are more closely related to the optical functions, are more easily accessible, and are of greater direct importance in the design of photovoltaic and other devices.

\section{ACKNOWLEDGMENTS}

The authors acknowledge helpful discussions with G. E. Jellison, Jr. on the development of the Tauc-Lorentz expressions. The authors acknowledge support of this research by the National Renewable Energy Laboratory under Subcontract Nos. XAF-8-17619-22 and AAD-9-18-668-09 and by the National Science Foundation under Grant Nos. DMR9820170 and DMR-0137240.

\footnotetext{
${ }^{1}$ Spectroscopic Ellipsometry, Proceedings of the Second International Conference, edited by R. W. Collins, D. E. Aspnes, and E. A. Irene (Elsevier, Amsterdam, 1997).

${ }^{2}$ R. E. I. Schropp and M. Zeman, Amorphous and Microcrystalline Silicon Solar Cells: Modeling, Materials and Device Technology (Kluwer, Boston, 1998).

${ }^{3}$ G. D. Cody, in Semiconductors and Semimetals, edited by J. I. Pankove (Academic, Orlando, FL, 1984), Vol. 21B, p. 11.

${ }^{4}$ D. E. Aspnes, A. A. Studna, and E. Kinsbron, Phys. Rev. B 29, 768 (1984).

${ }^{5}$ G. E. Jellison, Jr., Thin Solid Films 234, 416 (1993).

${ }^{6}$ A. S. Ferlauto, G. M. Ferreira, C. Chen, P. I. Rovira, C. R. Wronski,
} 
R. W. Collins, X. Deng, and G. Ganguly, in Photovoltaics for the 21st Century II, edited by R. D. McConnell and V. K. Kapur (Electrochemical Society, Pennington, NJ, 2001), p. 199.

${ }^{7}$ A. R. Forouhi and I. Bloomer, Phys. Rev. B 34, 7018 (1986).

${ }^{8}$ W. A. McGahan, T. Makovicka, J. Hale, and J. A. Woollam, Thin Solid Films 253, 57 (1994).

${ }^{9}$ T. Yamaguchi, Y. Kaneko, A. H. Jayatissa, M. Aoyama, A. V. Zotov, and V. G. Lifshits, J. Appl. Phys. 77, 4673 (1995).

${ }^{10}$ G. E. Jellison, Jr. and F. A. Modine, Appl. Phys. Lett. 69, 371 (1996); 69, 2137 (1996).

${ }^{11}$ J. Leng, J. Opsal, H. Chu, M. Senko, and D. E. Aspnes, Thin Solid Films 313-314, 132 (1998).

${ }^{12}$ A. S. Ferlauto, J. Koh, P. I. Rovira, C. R. Wronski, R. W. Collins, and G. Ganguly, J. Non-Cryst. Solids 266-269, 269 (2000).

${ }^{13}$ W. Luft and Y. S. Tsuo, Hydrogenated Amorphous Silicon Alloy Deposition Processes (Marcel Dekker, New York, 1993).

${ }^{14}$ Amorphous and Microcrystalline Semiconductor Devices: Optoelectronic Devices, edited by J. Kanicki (Artech House, Boston, MA, 1991).

${ }^{15}$ W. A. McGahan and J. A. Woollam, Mater. Res. Soc. Symp. Proc. 349, 453 (1994).

${ }^{16}$ G. E. Jellison, Jr., Thin Solid Films 313-314, 33 (1998).

${ }^{17}$ J. Tauc, R. Grigorovici, and A. Vancu, Phys. Status Solidi 15, 627 (1966).

${ }^{18}$ F. Wooten, Optical Properties of Solids (Academic, New York, 1972).

${ }^{19}$ R. W. Collins and K. Vedam, in Encyclopedia of Applied Physics, edited by G. L. Trigg (VCH, New York, 1995), Vol. 12, p. 285.
${ }^{20}$ W. B. Jackson, S. M. Kelso, C. C. Tsai, J. W. Allen, and S.-J. Oh, Phys. Rev. B 31, 5187 (1985).

${ }^{21}$ R. M. Dawson et al., Mater. Res. Soc. Symp. Proc. 258, 595 (1992).

${ }^{22} \mathrm{~J}$. Spanier and K. B. Oldham, An Atlas of Functions (Hemisphere, Washington, DC, 1987), p. 233

${ }^{23}$ J. Koh, Y. Lu, C. R. Wronski, Y. Kuang, R. W. Collins, T. T. Tsong, and Y. E. Strausser, Appl. Phys. Lett. 69, 1297 (1996).

${ }^{24}$ L. Jiao, I. Chen, R. W. Collins, C. R. Wronski, and N. Hata, Appl. Phys. Lett. 72, 1057 (1998).

${ }^{25}$ D. E. Aspnes, in Optical Properties of Solids: New Developments, edited by B. O. Seraphin (North-Holland, Amsterdam, 1976), p. 799.

${ }^{26}$ C. R. Wronski and D. E. Carlson, in Photoconversion of Solar Energy, Vol. 3; Clean Energy from Photovoltaics (Imperial College, London, 2001).

${ }^{27}$ J. Yang, A. Banerjee, and S. Guha, Appl. Phys. Lett. 70, 2975 (1997).

${ }^{28}$ J. Koh, Y. Lee, H. Fujiwara, C. R. Wronski, and R. W. Collins, Appl. Phys. Lett. 73, 1526 (1998).

${ }^{29}$ G. Tao, M. Zeman, and J. W. Metselaar, Sol. Energy Mater. Sol. Cells 34, 359 (1994).

${ }^{30}$ B. Sopori, J. Madjdpour, Y. Zhang, W. Chen, S. Guha, J. Yang, A. Banerjee, and S. Hegedus, Mater. Res. Soc. Symp. Proc. 557, 755 (1999).

${ }^{31}$ R. J. Koval, C. Chen, G. M. Ferreira, A. S. Ferlauto, J. M. Pearce, P. I. Rovira, C. R. Wronski, and R. W. Collins, Mater. Res. Soc. Symp. Proc. (in press).

${ }^{32}$ K. Mui and F. W. Smith, Phys. Rev. B 38, 10623 (1988). 